
Revue del'histoire des religions
Revue de l'histoire des religions
$1 \mid 2019$
Corps, ascèse et extinction dans l'histoire du bouddhisme (Inde, Corée, Japon)

\title{
Acclimater l'herméneutique philosophique en islam : Shabestarî, de la critique des méthodes exégétiques à la théorie de l'historicité du Coran
}

Introducing philosophical hermeneutics in Islam: Shabestarî, from the critique

of exegetical methods to the theory of the historicity of the Quran

\section{Constance Arminjon}

\section{OpenEdition}

\section{Journals}

Édition électronique

URL : https://journals.openedition.org/rhr/9437

DOI : 10.4000/rhr.9437

ISSN : 2105-2573

Éditeur

Armand Colin

Édition imprimée

Date de publication : 1 mars 2019

Pagination : 121-149

ISBN : 978-2-200-93230-5

ISSN : 0035-1423

Référence électronique

Constance Arminjon, « Acclimater l'herméneutique philosophique en islam : Shabestarî, de la critique des méthodes exégétiques à la théorie de l'historicité du Coran », Revue de l'histoire des religions [En ligne], 1 | 2019, mis en ligne le 01 janvier 2022, consulté le 06 janvier 2022. URL : http:// journals.openedition.org/rhr/9437 ; DOI : https://doi.org/10.4000/rhr.9437 


\section{Acclimater l'herméneutique philosophique en islam : Shabestarî, de la critique des méthodes exégétiques à la théorie de l'historicité du Coran}

En acclimatant l'herméneutique philosophique, le théologien shi'ite iranien Mohammad Mojtahed Shabestarî renouvelle de manière décisive les sources et l'orientation de la pensée religieuse musulmane. À rebours de durables tendances à l'apologétique, il illustre le tournant critique survenu au début des années 1990 dans l'histoire intellectuelle shi'ite. Cet article examine les rapports que le théologien établit avec la tradition islamique d'une part et avec la philosophie européenne d'autre part, et le double usage qu'il fait de l'herméneutique philosophique. Dans son premier ouvrage, Shabestarî a utilisé l'herméneutique dans un but essentiellement épistémologique. Plus récemment, il s'est appuyé sur une hypothèse herméneutique pour reconsidérer la nature et le statut du texte coranique.

\section{Introducing philosophical hermeneutics in Islam :}

Shabestarî, from the critique of exegetical methods to the theory of the historicity of the Quran

While introducing philosophical hermeneutics, the Iranian Shi'i theologian Mohammad Mojtahed Shabestarî has brought about a significant renewal in Islamic religious thought. Breaking with a longlasting apologetic trend, he epitomizes the critical turn that occurred in the early 1990s in the intellectual history of Shiite Islam. The article aims at analysing the theologian's stance towards Islamic tradition and European philosophy, and his manifold use of hermeneutics. In his first work, Shabestarî resorted to hermeneutics with an epistemological purpose. More recently he has worked from a hermeneutical assumption in order to reconsider the nature of the Quranic text. 
Depuis deux décennies, le théologien shi' ite iranien Mohammad Mojtahed Shabestarî (1936-) passe les disciplines du savoir islamique classique au crible de l'herméneutique philosophique. De la critique des méthodes exégétiques et théologiques à l'élucidation de l'historicité du texte coranique, il recourt à l'herméneutique en vue de refonder les rapports entre pensée religieuse et philosophie en islam. Car il est convaincu que le monde musulman ne pourra sortir des problèmes théoriques et pratiques engendrés par la civilisation moderne que s'il révise les fondements de l'exégèse et de la connaissance de la religion ${ }^{1}$. À rebours des tendances à l'apologétique qui ont marqué les générations précédentes, son cheminement illustre le tournant critique survenu au début des années 1990 dans l'histoire intellectuelle shi'ite et qui est aussi incarné par ${ }^{\mathrm{c}} \mathrm{Abd}$ ol-Karîm Sorûsh, Mohsen Kadîwar et d'autres². Son propos concorde également avec celui de plusieurs penseurs sunnites qui ont tenté de renouveler l'exégèse du Coran à partir de théories linguistiques ou de philosophies herméneutiques européennes - tel l'Égyptien Nasr Hâmid Abû Zayd et les théologiens de «l'École d'Ankara ${ }^{3}$ ». Toutefois Shabestarî se distingue des auteurs shi'ites par son exhaustivité méthodique. Il se démarque en outre des penseurs sunnites parce qu'il est un clerc éminent et qu'il opère une rigoureuse critique de sa propre tradition.

En acclimatant l'herméneutique, Shabestarî renouvelle de manière décisive les sources et l'orientation de la théologie

1. Mohammad Mojtahed Shabestarî, Hermenûtîk, ketâb o sonnat (farâyand-e

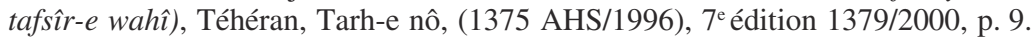
Le sigle AHS désigne le calendrier hégirien solaire en usage en Iran. Dans les références d'édition données ci-après, la première date est celle de ce calendrier.

2. Voir Said Amir Arjomand, "The Reform Movement and the Debate on Modernity and Tradition in Contemporary Iran », International Journal of Middle East Studies, 34/4, Nov. 2002, p. 719-731, spécialement p. 723-724.

3. L'expression désigne plusieurs membres de la Faculté de théologie d'Ankara qui recourent à l'herméneutique philosophique européenne dans leur exégèse du Coran. Voir Felix Körner, «Turkish Theology Meets European Philosophy: Emilio Betti, Hans-Georg Gadamer and Paul Ricœur in Muslim Thinking », Revista Portuguesa de Filosofia, T. 62, fasc. 2/4, 2006, p. 805-809; Rotraud Wielandt, "Main Trends of Islamic Theological Thought from the Late Nineteenth Century to Present Times ", in Sabine Schmidtke (éd.), The Oxford Handbook of Islamic Theology, Oxford, Oxford University Press, 2016, p. 707-764, ici p. 737-744. 
musulmane. Son œuvre a été peu étudiée jusqu'à présent. Certains ont examiné sa conception de la liberté et son rapport avec la philosophie kantienne ${ }^{4}$, d'autres sa nouvelle conception de la révélation ${ }^{5}$ et d'autres encore sa pensée juridique ${ }^{6}$. Par ailleurs, quelques chercheurs ont retracé sa biographie intellectuelle et étudié ses contacts avec la théologie chrétienne ${ }^{7}$. J'aimerais ici analyser les rapports que le théologien shi'ite établit avec la tradition islamique d'une part et avec la philosophie européenne d'autre part, et le double usage qu'il fait de l'herméneutique philosophique. Dans son premier ouvrage, Shabestarî a utilisé

4. Voir Roman Seidel, «Mohammed Schabestari, Iran. Die gottgefällige Freiheit », in Katajun Amirpur et Ludwig Ammann(éd.), Der Islam am Wendepunkt. Liberale und konservative Reformer einer Weltreligion, Freiburg (Allemagne), 2006 ; Idem, «Reading Kant in Teheran. Towards a Reception of the Iranian Reception of European Philosophy », Asiatische Studien / Études asiatiques, LXIV/3, 2010, p. 681-705 ; Idem, Kant in Teheran : Anfänge, Ansätze und Kontexte der Kantrezeption in Iran, Berlin, De Gruyter, 2014. Katajun Amirpur a traduit deux textes de Shabestarî et présente l'auteur dans Unterwegs zu einem anderen Islam, Freiburg am Brisgau, Herder, 2009. Elle résume brièvement la conception de la liberté du clerc iranien dans « "La ikraha fi 'l-din - There Is No Compulsion in Religion" - Or Is There ? », in Hans Georg Ziebert et Ernst Hirsch Ballin (éd.), Freedom of Religion in the $21^{\text {st }}$ Century, Leyde - Boston, Brill, 2016, p. 74-91.

5. Voir Ali Akbar, «A Contemporary Muslim Scholar's Approach to Revelation : Mohammad Mojtahed Shabestarî’s Reform Project », Arabica, 63/6, 2016, p. 656-680. Akbar étudie les conceptions de Shabestarî sur la révélation, mais n'analyse pas son rapport à l'herméneutique. Malgré le titre de son chapitre, Abbas Poya n'aborde que brièvement l'exégèse de Shabestarî dans « How the Prophet saw the world : On the Qur'ânic Exegesis of Mohammad Mojtahed Shabestari », in Abbas Poya et Farid Suleiman (éd.), Unity and Diversity in Contemporary Muslim Thought, Newcastle upon Tyne (G. B.), Cambridge Scholars Publishing, 2017, p. 208-227.

6. Ashk Dahlén, Islamic Law, Epistemology and Modernity. Legal Philosophy in Contemporary Iran, New York - Londres, Routledge, 2003, p. 163-185 ; Constance Arminjon Hachem, Les Droits de l'Homme dans l'islam shi' ite. Confluences et lignes de partage, Paris, Le Cerf, 2017, chap. 7, « Les droits de l'Homme au service de la foi. Herméneutique et philosophie pratique chez Mojtahed Shabestarî».

7. Yann Richard, «Un théologien chiite de notre temps, Mojtahed Shabestari », in Mohammad-Ali Amir-Moezzi, Meir M. Bar-Asher et Simon Hopkins (dir.), Le Shi'isme imamite quarante ans après. Hommage à Etan Kohlberg, Turnhout (Belgique), Brepols, 2009, p. 363-371 ; Mohsen Mottaghi, La Pensée chiite contemporaine à l'épreuve de la Révolution iranienne, Paris, L'Harmattan, 2012, p. 139-164. Sasan Tavassoli présente Shabestarî comme un « théologien existentialiste » dans le chapitre 5 de son livre Christian Encounters with Iran. Engaging Muslim Thinkers after the Revolution, Londres - New York, I. B. Tauris, 2011. 
l'herméneutique dans un but essentiellement épistémologique. Plus récemment, dans une série d'articles intitulée Lecture prophétique $d u$ monde $e^{8}$, il s'est appuyé sur une hypothèse herméneutique pour reconsidérer la nature et le statut du texte coranique.

\section{RAPPORT AUX SAVOIRS ISLAMIQUES CLASSIQUES ET À LA PHILOSOPHIE EUROPÉENNE MODERNE}

Il n'est pas opportun de présenter ici la biographie du théologien iranien. Mais il convient de rappeler qu'il est un éminent représentant du clergé shi'ite et qu'il a par ailleurs découvert de longue date la philosophie européenne moderne. Comme l'indique le titre de mojtahed habituellement ajouté à son nom, Shabestarî a suivi toutes les étapes de sa formation religieuse dans les séminaires de Qom (Iran), où il a été l'élève de maîtres majeurs de l'institution cléricale et le condisciple de plusieurs personnalités importantes du clergé actuel. C'est donc avec une compétence pleine et reconnue qu'il critique les savoirs qu'il a reçus. De surcroît, à la faveur d'une décennie de séjour en Allemagne - il fut recteur du Centre islamique de Hambourg de 1969 à 1978 -, il a étudié la philosophie européenne moderne et s'est profondément familiarisé avec les théologies protestante et catholique. Il a en particulier été très influencé par la critique herméneutique des textes sacrés du christianisme?

Convaincu de l'universalité de l'herméneutique philosophique, le théologien shi'ite ne s'interroge pas sur la pertinence de son utilisation en contexte islamique. «La critique n'est pas de nature à avoir des frontières », assure-t-il dans son premier ouvrage ${ }^{10}$. Dans le sillage des «savants de l'herméneutique» (hokamâ-ye hermenûtîk), Shabestarî se confronte à sa tradition en établissant avec elle un rapport si critique qu'il semble se placer à l'extérieur de celle-ci.

8. Shabestarî, Qerâ'at-e nabawî az jahân, inédit, diffusé sur le site de l'auteur entre 2008 et 2013 sous la forme d'une série d'articles. Cf. www. mohammadmojtahedshabestari.com.

9. Shabestarî présente ainsi sa biographie intellectuelle sur son site Internet, www.mohammadmojtahedshabestari.com.

10. Shabestarî, Hermenûtîk, ketâb o sonnat, p. 188. 


\section{Distanciation critique à l'égard de la tradition islamique}

Depuis les premiers contacts avec la pensée européenne moderne au $\mathrm{XIX}^{\mathrm{e}}$ siècle, des renouvellements ont été opérés dans le droit savant et dans la théologie politique shi'ites. Mais la théologie et les disciplines du savoir religieux n'ont pas été dans le même temps l'objet de cruciales mises en question au sein du clergé. De plus, des courants visant à la transformation de l'islam en idéologie se sont développés dans les années 1950. Prépondérants pendant plusieurs décennies, ils ont favorisé une appréhension apologétique de l'islam. À partir de la fin des années 1980, les bouleversements causés dans le droit, la théologie politique et les institutions religieuses par l'avènement d'un régime clérical en Iran ont fait naître le besoin d'un ample questionnement de la tradition shi'ite, besoin aiguisé par la crise des idéologies totalitaires et par la découverte plus large des philosophies européenne et américaine et des sciences humaines ${ }^{11}$. Avec d'autres clercs et penseurs laïcs, Shabestarî illustre l'apparition de ce nouveau paysage intellectuel qui se caractérise par un effort d'inventaire critique de l'héritage shi'ite et plus généralement islamique. Cependant l'inventaire n'est pas effectué avec les mêmes méthodes ni avec les mêmes sources par les uns et les autres.

La comparaison entre Shabestarî et le cheikh Mohsen Kadîwar (1959-) est à cet égard particulièrement éclairante. Comme son aîné, Kadîwar procède à une critique rigoureuse de sa tradition en vue de concevoir un islam adapté aux exigences de la modernité ${ }^{12}$. Il souligne également la nécessité d'historiciser les notions et les pratiques religieuses. Mais c'est de l'intérieur de sa propre tradition et en recourant seulement à des références internes qu'il développe sa critique du droit islamique et des évolutions

11. Cf. Mohsen Mottaghi, La Pensée chiite contemporaine à l'épreuve de la Révolution iranienne, op. cit. ; Constance Arminjon Hachem, Chiisme et État. Les clercs à l'épreuve de la modernité, Paris, CNRS Éditions, 2013, chap. 7.

12. Dans son ouvrage sur La Pensée politique en islam, Kadîwar retrace l'histoire du droit et de la théologie politique shi'ites. Cf. Andisheh-ye siyâsî dar eslâm, 3 vol., Vol. 1, Nazariyehhâ-ye dowlat darfeqh-e shîceh; Vol. 2, Hokûmat-e

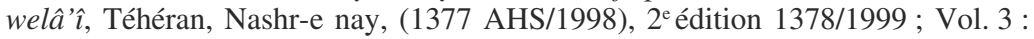
Hokûmat-e entesâbî̀, 1379-1380/2000-2001. Dans Le Droit des gens. L'islam et les droits de l'Homme, Kadîwar confronte l'héritage du droit islamique aux principes des droits de l'Homme. Voir Haqq al-nâs. Eslâm wa hoqûq-e bashar, Téhéran, Kavîr, (1387/2008), $4^{e}$ édition 1388/2009. 
de la théologie politique shi'ite. Au contraire Shabestarî suit une méthode élaborée dans une autre tradition de pensée pour instaurer un rapport critique avec l'ensemble de son héritage.

Cette distanciation est inaugurée dans son premier ouvrage publié en 1996 et se traduit dans la formulation même du titre : Herméneutique, Livre et Tradition. Par le seul choix d'un calque du terme étranger herméneutique (hermenûtîk en persan), Shabestarî marquait son refus d'employer le lexique islamique classique de l'exégèse - tafsîr (exégèse, interprétation), ta'wîl (exégèse, exégèse allégorique), sharh (commentaire), kashf al-mahjûb (dévoilement). Cependant le théologien ne se bornait pas à puiser à de nouvelles sources pour livrer sa propre interprétation des textes scripturaires de l'islam (le Livre - le Coran - et la Tradition). Il montrait en outre sa volonté de rupture par son propos. L'exégèse classique vise à mettre au jour des correspondances : de textes, de notions philosophiques avec des termes scripturaires, de structures de mondes, de structures théologiques, de degrés anthropologiques. Elle comprend plusieurs étapes: examen linguistique, analyse du lien du texte avec d'autres, explication du sens littéral et élucidation des significations spirituelles et philosophiques - herméneutique au sens ancien. L'exégèse shi'ite repose en outre sur le postulat que le privilège de l'exégèse juste appartient aux Imâms ${ }^{13}$. Dans cette configuration, le savant ne peut qu'expliciter les propos des Imâms. Dès lors, l'herméneutique classique consiste à introduire une interprétation neuve sans contrevenir aux traditions du prophète Muhammad et des Imâms. Or Shabestarî innovait en ne se situant pas sur le terrain de l'exégèse classique et en se plaçant dans un rapport d'extériorité critique à l'égard des pratiques herméneutiques de l'islam. Le théologien ne cherchait ni à discuter de la pertinence de l'herméneutique philosophique moderne ni à comparer les méthodes herméneutiques élaborées en islam et en Europe. Au lieu de livrer une exégèse philosophante des textes scripturaires, il préconisait une herméneutique des savoirs religieux de l'islam eux-mêmes. Il infléchissait ainsi radicalement la visée de l'exégèse et de la théologie en islam. En soumettant

13. Voir Mohammad-Ali Amir-Moezzi, Le Coran silencieux et le Coran parlant. Sources scripturaires de l'islam entre histoire et ferveur, Paris, CNRS Éditions, 2011. 
la théologie musulmane à une critique épistémologique et en promouvant l'élaboration d'une «nouvelle théologie » (kalâm-e jadîd) sur de nouvelles bases philosophiques, Sorûsh (1945-) adoptait dans les mêmes années une approche convergente ${ }^{14}$. Toutefois le philosophe iranien n'a procédé ni alors ni par la suite avec la même exhaustivité. Il n'a pas examiné méthodiquement les disciplines du savoir islamique dans leur diversité.

\section{Dans le sillage de la philosophie européenne}

Si la comparaison avec les contemporains de Shabestarî met en évidence le rapport singulier que le théologien entretient avec sa tradition, il faut comparer son attitude avec celle des générations précédentes pour saisir le changement historique qu'il introduit dans les rapports avec la philosophie européenne. Avec quelquesuns de ses contemporains, en particulier Sorûsh et Malekiyân (1956-), Shabestarî rompt délibérément avec la tendance apologétique qui prévalait jusqu'alors dans l'attitude des penseurs musulmans envers la philosophie européenne. La comparaison avec plusieurs penseurs éminents qui se sont confrontés à la pensée européenne est sur ce point significative et d'autant plus que Shabestarî se réfère à eux. Tout en visant à « la reconstruction de la philosophie religieuse musulmane» et en contribuant effectivement à la renouveler en mettant en avant la notion d'expérience religieuse, le penseur sunnite indien Muhammad Iqbal (1877-1938) adoptait à l'égard de la pensée européenne une position nettement apologétique ${ }^{15}$. En Iran, les clercs philosophes Mohammad Hosayn Tabâtabâ'î (1903-1981), dont Shabestarî fut l'élève, et Mortadâ Motahharî (1920-1979) critiquèrent la philosophie européenne moderne ${ }^{16}$. En Iraq, Muhammad Bâqîr

14. Voir 'Abd ol-Karîm Sorûsh, Qabd wa bast-e te'ûrîk-e sharî́at. Nazariyeh-ye takâmol-e macrefat-e dînî [Contraction et expansion théoriques de la Loi religieuse. Théorie de l'évolution de la connaissance religieuse], (1370/1991), Téhéran, Serât, $5^{\mathrm{e}}$ éd. hiver 1375/1996.

15. Muhammad Iqbal, The Reconstruction of Religious Thought in Islam (Londres, 1934), édition annotée par M. Saeed Sheikh, Lahore, Institute of Islamic culture, 1986, p. XXI-XXII.

16. Cf. Urs Gösken, «Perception of Western Modernity from the Gaze of Șadraism : Muḥammad Ḥusayn Ṭabāṭabā'̄̄'s and Murtaḍā Muṭahharī’s Critique of Modern Western Philosophy », International Journal of Persian Literature, vol. $1, \mathrm{n}^{\circ} 1,2016$, p. 142-163. 
al-Sadr (1935-1980) composa un ouvrage spécifique pour réfuter les philosophies modernes non musulmanes en vue de saper leur influence dans le monde musulman. Publié en 1959, Notre Philosophie (Falsafatunâ) a un sous-titre éloquent : Étude objective du champ du conflit intellectuel existant entre divers courants philosophiques et en particulier entre la philosophie islamique et le matérialisme dialectique (marxisme ${ }^{17}$ ). Si l'auteur vise principalement à récuser le marxisme, il consacre la première moitié du livre à «la théorie de la connaissance» et la seconde au « concept philosophique du monde». Il résume les principales épistémologies élaborées en Europe de Descartes à Marx et au positivisme, qu'il oppose en bloc à « la théorie de la connaissance dans notre philosophie ${ }^{18} »$.

À rebours de ces figures emblématiques, Shabestarî se place résolument dans le sillage de ceux qu'il appelle «les savants de l'herméneutique » européens. Pour justifier son ancrage dans la tradition herméneutique, il livre un constat catégorique et formule un avertissement. Selon lui, la pensée et les sociétés musulmanes traversent une crise à laquelle seule la critique et le renouvellement radical des méthodes d'appréhension de la religion permettront de trouver une issue ${ }^{19}$. D'œuvre en œuvre, le théologien soumet les disciplines du savoir et les doctrines théologiques et juridiques à une critique herméneutique. S'il cite régulièrement les théologiens chrétiens qui inspirent son approche - Barth, Tillich et Rahner principalement-, il ne se réfère pas toujours avec précision aux philosophes qui guident son travail. Dans son insistance didactique sur les a priori de la compréhension et de l'exégèse, il consonne singulièrement avec Heidegger et Gadamer. Mais force est de reconnaître l'éclectisme avec lequel il procède. Cet éclectisme apparaît de manière particulièrement nette dans son avant-dernier texte - Lecture prophétique du monde -, dans lequel Shabestarî s'appuie à la fois sur des tenants de l'herméneutique - Heidegger, Gadamer et Ricœur-, sur «les philosophes du

17. Muhammad Bâqir al-Sadr, Falsafatunâ. Dirâsat mawdû̀'iyya fì mu'tarak al-sirấc al-fikrî al-qâ'im bayna mukhtalif al-tayyârât al-falsafiyya wa khâssatan al-falsafat al-islâmiyya wa al-mâddiyyat al-diyâliktîkiyya (al-mârksiyya), (1959), Beyrouth, Dâr al-Tacâruf, 2édition révisée 1998.

18. al-Sadr, Falsafatunâ, p. 141-145.

19. Shabestarî, Hermenûtîk, ketâb o sonnat, p. 9. 
langage » dont John Langshaw Austin, sur Wittgenstein ou encore sur Saussure. Suivant son propos, que l'on soit «structuraliste» comme Saussure et d'autres, ou adepte de l'herméneutique (ahl-e hermenûtîk) comme Heidegger, Wittgenstein et Austin, lorsque l'on est en présence d'un texte en langage humain et que l'on est dans une situation de compréhension, on se trouve face à quelqu'un qui «donne un message », est «partie d'un dialogue » ou «acteur du discours ${ }^{20} »$. Nous reviendrons plus loin sur la conception du texte coranique que Shabestarî élabore dans son ouvrage.

\section{De la CRitique aVANT toute Chose : ÉPISTÉMOLOGIE DE L'EXÉGÈSE ET DES DISCIPLINES DU SAVOIR RELIGIEUX}

Conscient de la nouveauté de sa démarche dans les milieux savants musulmans, Shabestarî procède de manière didactique. Dans son premier ouvrage, il présente d'abord l'herméneutique philosophique elle-même. Puis il en met en œuvre les enseignements et se livre à une épistémologie de l'exégèse, du droit savant et de la théologie. Il propose enfin sa propre méthodologie en appelant à une critique multiforme et incessante.

\section{Introduction à l'herméneutique}

Les deux premiers chapitres de Herméneutique, Livre et Tradition peuvent être considérés comme une introduction à l'herméneutique. Shabestarî retrace l'émergence de l'herméneutique philosophique en Europe et décrit longuement ses principes. Sans se référer à des œuvres précises, le clerc livre une historiographie du renouvellement de l'exégèse. Selon lui, l'idée que la compréhension est une sorte de connaissance qui a sa propre méthode est apparue au $\mathrm{XIX}^{\mathrm{e}}$ siècle. "Jusqu'alors on ne parlait que des règles de l'exégèse des textes religieux,

20. Shabestarî, Qerâ'at-e nabawî̀ az jahân, "Fahmîdan-e ensân yâ fahmândan-e khodâ? " [Est-ce l'homme qui comprend ou Dieu qui fait comprendre ?], nº, 15 tîr 1389 / 6 juillet 2010. Le texte est diffusé sous la forme d'un fichier numérique sans pagination. 
philosophiques, artistiques et juridiques et dans ces domaines il $\mathrm{y}$ avait des questions et des antécédents ${ }^{21}$. » D'après lui, la prise de conscience de l'historicité de la compréhension elle-même a entraîné le développement progressif de l'herméneutique telle qu'elle existe aujourd'hui. Shabestarî met en avant la dimension critique de l'exégèse et en discerne trois aspects. Premièrement, un texte n'est correctement compris que par une exégèse. Deuxièmement, tout texte est susceptible de plusieurs exégèses et « la compréhension du sens du texte n'est pas une question simple». Troisièmement, il faut identifier le sens correct du texte et écarter les exégèses non pertinentes ${ }^{22}$. En se référant aux "savants de l'herméneutique », Shabestarî dévoile ensuite les "prolégomènes» (moqaddamât) de tout processus d'exégèse. Quoiqu'il ne cite ni ne mentionne aucun auteur, son analyse évoque Heidegger et Gadamer dont il reprend presque terme pour terme le lexique. Il énumère ainsi et explique les notions de " compréhensions préalables (pîshfahmhâ) et savoirs préalables (pîshdânesteh)» de l'exégète ; les intérêts ('alâyeq) et les attentes (entezârât) qui orientent l'exégète; les questions de l'exégète à propos de l'histoire; le discernement du centre du sens du texte (kashf-e markaz-e macnâ-ye matn); "la traduction du texte dans l'horizon historique de l'exégète » (tarjomeh-ye macnâ-ye matn dar afaq-e târîkhî-e mofasser ${ }^{23}$ ).

De l'exégèse, le théologien généralise à l'ensemble des savoirs et constate que tout effort scientifique est mené à partir de compréhensions préalables et spécifiques à chaque discipline. Aussi ne faut-il pas confondre les visées des différentes sciences et chercher par exemple dans les textes sacrés des réponses à des questions des sciences expérimentales ${ }^{24}$. D'autre part, tout en soulignant la nécessité de déceler l'historicité des textes, Shabestarî se défend d'une conception historiciste : «Il faut admettre qu'en dépit de tous les importants changements historiques qui ont rendu différentes nos expériences et celles des hommes du passé, des expériences fondamentales concernant le fondement et la base de l'humanité nous sont communes avec les hommes du passé. C'est

21. Shabestarî, Hermenûtîk, ketâb o sonnat, p. 13.

22. Shabestarî, Hermenûtîk, ketâb o sonnat, p. 16.

23. Shabestarî, Hermenûtîk, ketâb o sonnat, p. 17-31.

24. Shabestarî, Hermenûtîk, ketâb o sonnat, p. 24. 
à l'ombre de ces choses communes que nous reconnaissons nos questions dans les leurs ${ }^{25}[\ldots] »$.

Après avoir exposé la méthode de l'analyse herméneutique, Shabestarî avance le principe, capital dans sa doctrine théologique, suivant lequel la religiosité est tributaire des connaissances humaines de son temps. Dans le second chapitre, il met en lumière la part humaine et partant historique de l'expérience religieuse. Or le caractère humain et l'historicité des savoirs ne se répercutent pas seulement sur les doctrines théologiques, mais aussi sur l'exégèse et sur les prescriptions juridiques. À la suite de cette leçon, le théologien procède à une analyse herméneutique des principales disciplines du savoir classique musulman.

\section{Herméneutique de l'exégèse juridique}

En raison de l'importance historique du droit savant ( $f i q h)$ dans l'organisation des sociétés musulmanes et sans doute aussi parce qu'il vit dans un régime fondé sur la souveraineté du savant juriste (velâyat-e faqî $h^{26}$ ), Shabestarî critique exhaustivement cette discipline et ses méthodes.

À l'appui de la notion de compréhensions préalables, le clerc montre que le droit savant musulman repose sur des postulats externes à sa discipline ${ }^{27}$. Le savant juriste (faqîh), observe-t-il, a toujours besoin de compréhensions préalables pour rendre un avis de droit religieux, lesquelles ne relèvent pas de la science du fiqh. Les musulmans différencient unanimement des préceptes stables et des préceptes changeants dans l'islam, mais personne ne peut déterminer quels préceptes du Coran ou de la Tradition sont stables et lesquels sont changeants. « Or l'ijtihâd et la consultation en droit religieux n'ont pas de sens si cette question n'est pas clarifiée ${ }^{28}$. »

25. Shabestarî, Hermenûtîk, ketâb o sonnat, p. 31 .

26. Formulée par Khomaynî dans un traité intitulé La guidance du savant juriste. Le gouvernement islamique (Velâyat-e faqîh. Hokûmat-e eslâmî, 1970), la théorie de la souveraineté du juriste religieux justifie la dévolution de toutes les fonctions d'autorité de l'Imâm à un juriste religieux. Elle fut institutionnalisée dans la Constitution de la République islamique d'Iran adoptée en 1979.

27. Shabestarî, Hermenûtîk, ketâb o sonnat, chap. 3 : «Compréhensions préalables des savants juristes, éternité des préceptes de la religion et discernement des questions ».

28. Shabestarî, Hermenûtîk, ketâb o sonnat, p. 40. 
Shabestarî examine ensuite longuement la question de l'historicité des préceptes car elle a été une préoccupation majeure des penseurs musulmans contemporains ${ }^{29}$. D'après le théologien, les juristes religieux doivent entreprendre un «ijtihâd nouveau » qui s'appuie sur des connaissances qui relèvent de la philosophie et des sciences humaines et non du droit savant et de sa méthodologie. L'humanité ne peut qu'être tributaire des connaissances de son temps: « $\grave{A}$ chaque époque, nous devons rectifier les compréhensions préalables à ce que l'on déduit du Coran et de la Tradition, et ce travail n'est possible que par la révision des savoirs et connaissances de chaque époque ${ }^{30} »$. D'après le théologien, du fait que les écoles religieuses se sont tenues à l'écart des sciences humaines, il n'y a pas aujourd'hui en islam de philosophie du droit ni de philosophie morale, politique ou économique.

À l'encontre d'un courant de pensée qui revendique l'exhaustivité du droit savant islamique, Shabestarî veut circonscrire le champ d'extension du fiqh en recourant à une approche épistémologique ${ }^{31}$. En particulier, à rebours d'un vaste mouvement qui commença dans les années 1950 et qui culmina, dans le monde shi'ite, avec l'adoption de la Constitution de la République islamique d'Iran à l'automne 1979, il met en question la possibilité de déduire des préceptes politiques à partir des textes scripturaires ${ }^{32}$. Pour justifier sa délimitation du champ d'application du droit savant, Shabestarî use de plusieurs arguments. Il montre d'abord que l'historicité affecte non seulement les doctrines et les institutions, mais aussi la constitution même de la discipline du droit savant. Il inventorie également les conceptions élaborées par les ulémas à propos du contenu politique des sources scripturaires de l'islam, en insistant sur le fait qu'elles sont sous-tendues par des compréhensions et des attentes théologiques et anthropologiques préalables $^{33}$. Pour résoudre

29. Chap. 4 : «Compréhensions préalables des savants juristes et permanence ou changement des objets des préceptes de la religion ».

30. Shabestarî, Hermenûtîk, ketâb o sonnat, p. 51.

31. Chap. 5: «Avec la science du droit savant on ne peut organiser des institutions, on peut seulement répondre à certaines questions ».

32. Chap. 6, «Avis et avis juridiques des savants juristes en matière politique », et chap. 7, "Compréhensions préalables, intérêts et attentes des savants juristes et questions de société et de gouvernement ».

33. Shabestarî, Hermenûtîk, ketâb o sonnat, p. 76. 
les problèmes d'aujourd'hui, Shabestarî préconise d'élaborer une philosophie et une épistémologie nouvelles : "l'évolution du droit savant doit commencer par la transformation des fondements philosophiques et épistémologiques de la conception de la religion et de l'anthropologie du savant juriste ${ }^{34} »$. La philosophie et l'épistémologie nouvelles ne seront jamais des substituts de religion mais serviront de fondations à la religion de l'âge moderne. Il faut prendre en considération le fait que les religions révélées sont confrontées depuis quelques siècles à des philosophies et sciences nouvelles ${ }^{35}$.

Suivant la méthode qu'il a adoptée à propos du domaine politique, Shabestarî met au jour les limites du droit savant en matière économique ${ }^{36}$. Il entend ici rouvrir le débat sur les implications économiques du droit savant, qui était né à la fin des années 1950 dans un contexte de réaffirmation de la place du fiqh dans le droit étatique. Plus précisément, il commente l'œuvre de Muhammad Bâqir al-Sadr consacrée à l'économie. Dans Notre économie (Iqtisâdunâ) publié en 1960, le clerc iraquien conçut les principes d'une économie spécifiquement islamique. Selon Shabestarî, al-Sadr chercha à dégager des préceptes du Coran et de la Tradition et à les ériger en principes juridiques. Or, souligne le théologien iranien, on ne peut déduire quoi que ce soit des textes scripturaires - ni d'aucun texte - sans idées préalables. C'est pourquoi Muhammad Bâqir al-Sadr et ceux qui partageaient son point de vue ont interprété certains versets dans un sens que d'autres exégètes ne leur donnaient pas. C'est avec une «mentalité d'homme du $\mathrm{XX}^{\mathrm{e}}$ siècle » qu'al-Sadr a fait une exégèse économique du Coran et de la Tradition ${ }^{37}$. En somme, conclut Shabestarî, on ne peut élaborer de droit savant économique sans avoir au préalable élaboré une philosophie du droit et une philosophie politique et morale ${ }^{38}$.

Ayant mis au jour le processus de l'ijtihâd et les postulats sousjacents à la discipline du droit savant, puis démontré les limites

34. Shabestarî, Hermenûtîk, ketâb o sonnat, p. 88.

35. Shabestarî, Hermenûtîk, ketâb o sonnat, p. 91.

36. Cf. Chap. 11, «Les compréhensions préalables des savants juristes et leurs avis juridiques économiques ».

37. Shabestarî, Hermenûtîk, ketâb o sonnat, p. 147-148.

38. Shabestarî, Hermenûtîk, ketâb o sonnat, p. 150-156. 
épistémologiques du fiqh, le théologien parachève son effort critique en appelant à une révision du droit savant à la lumière des sciences humaines modernes ${ }^{39}$. D'après lui, les «résultats » de la philosophie et des sciences humaines peuvent servir «d'outil de critique et de décantation des compréhensions préalables qui sous-tendent l'ijtihâd théologique et juridique ». Cela permet de délimiter judicieusement les domaines relevant de la religion et ceux relevant de la science. Une fois que les principes éthiques qui régissent les sociétés ont été définis, il appartient aux spécialistes de politique, d'économie et d'affaires sociales de définir les textes constitutionnels et les politiques générales ${ }^{40}$. Or cela requiert «un climat de liberté d'expression tant pour les opposants que pour les partisans ${ }^{41} »$.

\section{Herméneutique de l'exégèse théologique}

À la lumière de l'herméneutique philosophique, l'auteur veut montrer que les divergences d'exégèse qui divisent les théologiens musulmans dérivent des hypothèses et données préalables sur lesquelles leurs exégèses reposent ${ }^{42}$. Pour commencer, il classifie les types de compréhension du Coran et de la Tradition. Reprenant une typologie traditionnelle, il distingue quatre groupes: les Gens du hadîth ${ }^{43}$, les ash ${ }^{c}$ arites et les premiers musulmans (salafiyeh); les théologiens muctazilites; les philosophes et enfin les mystiques ('orafâ). "Chacune de ces compréhensions a son propre "horizon exégétique". Dans chaque horizon, il y a une série de compréhensions préalables et de postulats ». Les horizons sont aussi appelés «climats » (fad $\underline{a})$. Le climat de compréhension de la première catégorie est un climat d'écoute et de soumission.

39. Cf. Chap. 8, «Les théories et avis juridiques religieux sont critiquables, participation de tous à la formation des théories et des avis juridiques, nécessité d'un libre examen et d'une libre critique en matière de religion ».

40. Shabestarî, Hermenûtîk, ketâb o sonnat, p. 94-96.

41. Shabestarî, Hermenûtîk, ketâb o sonnat, p. 96.

42. Cf. chap. 9 «Compréhensions préalables des principaux exégètes de la Révélation musulmane, des Gens du hadîth et des ash ${ }^{c}$ arites aux muctazilites, aux gnostiques et aux récents exégètes tel le défunt Tabâtabâ'î̀ ».

43. Littéralement, "partisans des traditions». L'expression désigne les personnes qui se sont spécialisées dans la collecte et l'étude des traditions du prophète Muhammad. Cf. Joseph Schacht, «Ahl al-Hadîth», Encyclopédie de l'islam. Nouvelle édition, édition en ligne. 
Dans celui de la seconde, la réflexion abstraite prévaut ( $t a^{c} a q q o l-e$ $e^{c}$ tezâlî). Le climat de compréhension de la troisième catégorie se distingue par le recours à l'intelligence philosophique, qui diffère du précédent type de réflexion. Enfin, le climat de la quatrième catégorie est un climat marqué par la souffrance de l'exil et par le désir de retour à l'origine, par l'intériorité et l'union avec l'existence ${ }^{44}$.

D'après Shabestarî, les Gens du hadîth qui vivaient au temps du prophète Muhammad acceptaient ce que disait celui-ci «sans réflexion rationnelle approfondie». Leur attitude était due au charisme exceptionnel du Prophète ${ }^{45}$. L'auteur essaie de définir le charisme des prophètes en général et de Muhammad en particulier. Il se réfère aux travaux du théologien allemand Rudolf Otto (1869-1937) et notamment à son ouvrage Das Heilige.

Suivant à peu près la même typologie, Shabestarî examine ensuite les conceptions du statut de la parole révélée ${ }^{46}$. Il distingue les doctrines ash'arites et muctazilites; la conception spéciale d'Ibn Kullâb, théologien du III $^{\mathrm{e}}$ siècle de l'Hégire ; les doctrines des philosophes et la doctrine mystique d'Ibn ${ }^{\mathrm{c}} \mathrm{Arabî} \mathrm{(m.} \mathrm{1240).} \mathrm{Or,}$ par-delà la divergence qui les sépare, philosophes et théologiens musulmans partagent l'idée selon laquelle «le contenu de la "parole de Dieu révélée" est un ensemble d'informations sur le monde de l'existence ». D'après cette approche, l'exégèse du Coran consiste à obtenir ces informations et significations ${ }^{47}$. Shabestarî contestera longuement cette conception dans sa Lecture prophétique du monde. Pour lui, Ibn ${ }^{\mathrm{c} A r a b i ̂ ~ s e m b l e ~ a v o i r ~ e ́ t e ́ ~ l e ~}$ premier à avoir une autre vue sur le sens de la parole révélée. En effet, il a envisagé la «continuité de la Révélation » (estemrâr-e wahî). Il a divisé la prophétie en législation d'une part et nonlégislation d'autre part. De plus, il a mis le caractère révélé de la parole en relation avec la manière dont les hommes reçoivent la parole. La parole révélée se distingue des autres paroles en ce qu'elle est perçue comme une «tout autre parole ${ }^{48} »$. Acceptée

44. Shabestarî, Hermenûtîk, ketâb o sonnat, p. 103.

45. Shabestarî, Hermenûtîk, ketâb o sonnat, p. 104.

46. Cf. chap. 10 «Sens différents de la "parole révélée" chez les sages divinisés [mota'allehân] ».

47. Shabestarî, Hermenûtîk, ketâb o sonnat, p. 139-140.

48. Shabestarî, Hermenûtîk, ketâb o sonnat, p. 140. 
par de nombreux mystiques musulmans, la conception d'Ibn ${ }^{\mathrm{c}} A$ rabî peut être comparée à celles de certains théologiens protestants modernes tels que Karl Barth et Paul Tillich ${ }^{49}$.

\section{Méthodologie de la nouvelle théologie}

Dès son ouvrage inaugural, Shabestarî articule son épistémologie des savoirs religieux islamiques à des propositions destinées à refonder la théologie. Les chapitres XIV, XV et XVI s'apparentent à un Discours de la méthode de la compréhension des textes et de la théologie. L'auteur promeut une démarche triplement critique : recours à la critique historique des textes (chapitre XIV); critique de la pensée théologique traditionnelle en vigueur dans l'islam contemporain (chapitre XV) ; insistance sur la nécessité de critiquer sans cesse les doctrines religieuses (chapitre XVI).

Pour faire valoir la nécessité de renouveler les méthodes d'appréhension de la religion en islam, Shabestarî se réfère fréquemment aux transformations survenues dans le christianisme. C'est le cas par exemple lorsqu'il rappelle que la méthode historico-critique a été d'abord appliquée aux textes religieux du christianisme. Pour justifier la nécessité d'intégrer la méthode historico-critique dans la méthodologie de l'exégèse en islam, Shabestarî présente de manière schématique la méthode critique. Il passe sous silence la diversité des méthodes historiques élaborées ainsi que les débats sur l'historicisme et la critique herméneutique de l'historicisme.

«Finalement, cette méthode a été appliquée à l'étude des livres sacrés du christianisme [...] La théologie chrétienne moderne est l'un des fruits de ce face-à-face. Aujourd'hui, dans la théologie chrétienne, on s'efforce d'interpréter les trois questions importantes que sont la révélation, les miracles et l'apparition de Dieu en vue du salut de l'humanité, d'une manière qui ne contredise pas la vision du monde et la méthode de la critique historique. Des conceptions théologiques importantes et intéressantes sont apparues dans cette exégèse nouvelle. Et l'on peut dire que durant

49. Shabestarî, Hermenûtîk, ketâb o sonnat, p. 144. 
les deux derniers siècles la théologie chrétienne a connu une évolution fondamentale ${ }^{50} »$.

Outre le recours à la critique historique, Shabestarî préconise de «faire une évaluation générale de la pensée théologique traditionnelle de l'islam contemporain ${ }^{51}$. » Avant de procéder à cette évaluation, il précise ce qu'il entend par critique (naqd) : il entend «évaluer l'intelligibilité ou l'inintelligibilité des méthodes et des questions théologiques usuelles parmi les musulmans eu égard aux questions qui se posent aujourd'hui dans le monde autour de la religion et de la religiosité. La pensée théologique traditionnelle de l'islam contemporain est-elle intelligible pour faire face à ces questions? En second lieu, la critique de la théologie ou de la philosophie islamique ne signifie pas la critique de la religion musulmane ${ }^{52}$. »

L'auteur examine ensuite les fonctions remplies par la théologie en islam au cours de l'histoire. Selon lui, la théologie a rempli trois principales fonctions : exposer les fondements de la croyance et de la foi musulmane ; prouver les croyances ; «répondre aux soupçons venant de l'extérieur de la religion ${ }^{53} »$.

Shabestarî énumère les questions posées par la théologie musulmane classique: existence ou non-existence de Dieu; essence et attributs de Dieu ; nature de la Prophétie ; Muhammad était-il prophète ?; qu'est-ce que la vie future ? Ces questions visent à connaître une série de réalités sur Dieu, sur les créatures, l'Homme et sa destinée. "Dans un tel climat de pensée, l'incertitude et le scepticisme sont exceptionnels. »C'est dans un tel climat que la théologie musulmane vise à formuler des croyances absolument vraies. En d'autres termes, il n'y a pas vraiment de place pour le questionnement ${ }^{54}$. La théologie musulmane se caractérise aussi par l'idée que les questions de foi sont démontrables par la raison. "La théologie musulmane a été construite et s'est développée avec ces deux particularités. Mais à notre époque, le climat de la pensée a changé et toute question

50. Shabestarî, Hermenûtîk, ketâb o sonnat, p. 181-182.

51. Shabestarî, Hermenûtîk, ketâb o sonnat, p. 183.

52. Shabestarî, Hermenûtîk, ketâb o sonnat, p. 183.

53. Shabestarî, Hermenûtîk, ketâb o sonnat, p. 184-185.

54. Shabestarî, Hermenûtîk, ketâb o sonnat, p. 186, 187. 
part [de ce changement]. Dans le climat de pensée nouveau, il n'y a plus de place pour le dogmatisme philosophique ni scientifique $^{55}[\ldots] »$

Dans le nouveau climat de pensée, «la méthode de démonstration»n'est pas adéquate. Il faut donc accorder la fonction et les principes de la théologie au climat nouveau: «Dans un tel climat, la théologie ne peut plus avoir la structure et les particularités qu'elle avait auparavant ni rechercher les buts qui étaient auparavant les siens [...] On s'interroge aujourd'hui prioritairement sur le rôle de la religion et non sur la conformité ou la non-conformité des prédicats religieux [gozârehhâ] avec le

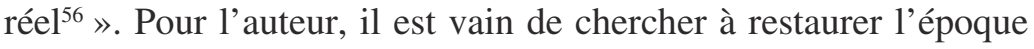
du dogmatisme philosophique : «Nous qui sommes pieux faisons face à une bifurcation : [... : prétendre restaurer le climat de pensée du dogmatisme ou bien élaborer des notions et une méthode nouvelles pour aborder la religion] [...] Nous ne pouvons choisir que la seconde voie, nous n'en avons pas d'autre ${ }^{57}$. »

Shabestarî souligne que le climat de pensée en Iran n'est pas fermé, puisque la philosophie occidentale, les sciences sociales, l'épistémologie et d'autres disciplines y sont enseignées. Les étudiants des écoles religieuses eux-mêmes vont en Occident pour se former à la philosophie et aux sciences occidentales. Les écoles religieuses doivent se confronter à bras-le-corps aux questions nouvelles ${ }^{58}$.

La démarche herméneutique incite enfin Shabestarî à préconiser une critique incessante des doctrines religieuses ${ }^{59}$. En préambule du chapitre XVI, l'auteur rappelle qu'il a déjà précisé le sens qu'il donnait au terme de critique ${ }^{60}$. Il insiste sur le fait qu'il faut comprendre ce terme correctement: la critique consiste à examiner tous les aspects d'une pensée ou d'une affirmation ainsi que ses conséquences inévitables. Parce que nous sommes des êtres limités, relatifs, inscrits dans le temps et l'espace, nos

55. Shabestarî, Hermenûtîk, ketâb o sonnat, p. 187, 188.

56. Shabestarî, Hermenûtîk, ketâb o sonnat, p. 189.

57. Shabestarî, Hermenûtîk, ketâb o sonnat, p. 192.

58. Shabestarî, Hermenûtîk, ketâb o sonnat, p. 192.

59. Cf. le chapitre 16, « Pourquoi faut-il critiquer la pensée religieuse ?».

60. Hermenûtîk, ketâb o sonnat, p. 193. 
critiques le sont aussi ${ }^{61}$. Après avoir énoncé cette maxime inspirée par l'herméneutique, Shabestarî discerne la spécificité de la «pensée religieuse ». Il invite à distinguer la « pensée religieuse » des «connaissances scientifiques et philosophiques ». La pensée religieuse englobe toute l'existence de l'Homme, ce qui n'est pas le cas des sciences ni de la philosophie. Car la pensée religieuse a une portée existentielle ${ }^{62}$.

L'auteur développe ensuite sa conception sur la nécessité de la critique, tout en précisant que celle-ci a plusieurs dimensions. La critique de la pensée religieuse doit être menée tantôt de l'extérieur, tantôt de l'intérieur. Ces deux formes de critique se différencient par le fait que «[...] la critique de l'extérieur n'est pas croyante, ou à tout le moins le critique ne se place pas dans une position de foi ». Comme exemples de critiques extérieures, Shabestarî cite les critiques de la religion faites par Feuerbach, Marx, Sartre, Freud et Russell, en ajoutant que leurs critiques étaient faites à partir d'une position qui n'était pas seulement hors de la foi mais contre la foi $^{63}$. La critique de la pensée religieuse faite de l'intérieur part au contraire d'une position de foi. Shabestarî indique que l'étude qu'il mène dans son ouvrage s'inscrit dans cette perspective. Il avertit que l'un des problèmes de la société contemporaine consiste dans la confusion entre les deux formes de critique.

Dans l'islam, la critique de la pensée religieuse ne peut se faire que «sur l'axe de l'unicité divine, qui est le fondement de la foi musulmane ». Or au fil du temps la pensée de l'unicité « [...] a été affectée par la pétrification [tahajjor] ». «La pensée monothéiste au sens que nous avons donné, poursuit l'auteur, est une sorte de relation entre l'Homme et Dieu». Dans cette relation, l'Homme doit avoir toute sa place ${ }^{64}$. Cette conviction sera le motif principal $\mathrm{du}$ quatrième livre de Shabestarî, Réflexions sur une lecture humaine de la religion (Ta'ammolâtî dar qerâ'at-e rasmî az dîn, 2004).

Dans toute sa richesse, l'héritage de la pensée religieuse musulmane montre qu'il y a eu tout au long de l'histoire une critique de la pensée religieuse, dans un climat «semi-libre sinon

61. Hermenûtîk, ketâb va sonnat, p. 194.

62. Hermenûtîk, ketâb va sonnat, p. 194-195.

63. Hermenûtîk, ketâb va sonnat, p. 195.

64. Hermenûtîk, ketâb va sonnat, p. 196-198. 
libre ». En conséquence l'auteur invite à poursuivre cet effort : « Il faut prendre en considération le fait que la critique interne de la pensée religieuse est bénéfique pour la foi et la religiosité. Il faut ouvrir l'espace pour cette critique ${ }^{65}$. » Cependant la critique interne ne peut se faire dans l'ignorance des critiques extérieures.

Avec prudence, Shabestarî précise que la critique interne de la pensée religieuse «n'est pas incompatible avec» le respect des principes de la foi ni avec le respect des savants religieux. Pour l'auteur, une société dans laquelle il n'y a pas de critique de la religion et de la foi n'est pas propice pour les croyants. De surcroît, la foi ne donne pas de manière définitive un contenu stable (thâbet) de connaissances ${ }^{66}$.

Rompant avec les pratiques exégétiques classiques, Shabestarî a d'abord recouru à l'herméneutique philosophique pour critiquer non seulement la discipline de l'exégèse, mais plus largement l'ensemble des disciplines du savoir religieux. Dans Lecture prophétique du monde, la méthode herméneutique remplit une autre fonction : elle fournit une hypothèse permettant à l'auteur de réexaminer la nature du texte coranique. Une nouvelle fois, mais dans un autre sens, Shabestarî inverse la visée de la discipline de l'exégèse.

\section{LA COMPRÉHENSION DU TEXTE CORANIQUE ET SES IMPLICATIONS}

À la différence des quatre premiers livres de Shabestarî́ ${ }^{67}$, la Lecture prophétique du monde est un ouvrage inédit. Invoquant la censure et les critiques auxquelles il a été confronté après avoir fait paraître un article sur le phénomène de la révélation ${ }^{68}$, le théologien a décidé de ne pas publier la suite des textes qu'il a consacrés à cette question. Il les a seulement diffusés sur son site

65. Hermenûtîk, ketâb o sonnat, p. 200.

66. Hermenûtîk, ketâb o sonnat, p. 202-204.

67. Outre Hermenûtîk, ketâb o sonnat étudié ici, l'auteur a publié Îmân wa âzâdî (Foi et Liberté, 1997), Naqdî bar qerâ'ât-e rasmî az dîn. Bohrânhâ, châleshhâ, râhhallhâ (Critique d'une lecture officielle de la religion. Crises, défis et solutions, 2000), Ta'ammolâtî dar qerâ'at-e ensânî az dîn (2004, voir plus haut).

68. Intitulé Qerâ'at-e nabawî az jahân, l'article parut dans le numéro 6 de la revue Madreseh publié en 2007. 
Internet personnel. Ces textes ont été mis en ligne régulièrement entre février 2008 et août 2013 et sont présentés par l'auteur comme une série de quinze articles regroupés sous le même titre.

\section{Considérer la parole coranique d'un point de vue philosophique}

Quoiqu'il qualifie parfois son étude d' «exégèse libre» du Coran, le théologien n'élabore pas un commentaire du texte sacré. Dans le premier article de la série, il indique d'emblée qu'il ne vise pas à comprendre le sens du Coran, mais à savoir ce que disait le prophète de l'islam sur la parole coranique. En d'autres termes, Shabestarî entend élucider la nature du texte sacré de l'islam. Sans détour, il avance sa thèse. Suivant ses propos, en examinant le texte coranique comme un document historique, on note que les paroles du Coran sont celles d'un être humain bien qu'elles aient une source divine : «Ce prophète n'a pas dit que ce texte n'était pas sa parole ». En énonçant une telle affirmation, l'auteur est pleinement conscient de mettre en question le dogme de l'origine divine du Coran. C'est pourquoi il présente sa thèse comme la conclusion d'un questionnement herméneutique. Selon lui, si l'on considère les changements survenus dans le Hijâz et l'apparition de la civilisation musulmane, il faut se demander si l'on peut admettre que ces réalités aient résulté de paroles simplement récitées par un être humain. Pour clarifier cette question, assure l'auteur, il faut la considérer d'un point de vue philosophique et s'interroger sur le phénomène de la compréhension des paroles coraniques prononcées par le prophète Muhammad. À cet égard, il faut d'abord s'appuyer sur une définition du langage. Shabestarî se réfère à celle du philosophe allemand Albert Keller ${ }^{69}$, qu'il juge éclairante, suivant laquelle le langage repose sur cinq «axes»: un locuteur, un interlocuteur, un contexte, un peuple parlant une langue et un contenu. Or, poursuit le théologien, cette définition acceptée par la plupart des philosophes du langage montre que le langage est un phénomène humain qui a plusieurs composantes nécessaires. Sur ce point, il renvoie sans précision à Wittgenstein et au commentaire de celui-ci par Austin. Puis il ajoute qu'un locuteur véritable ne peut être qu'une personne douée d'esprit,

69. L'auteur se réfère à Albert Keller, Sprachphilosophie, 1979. 
dont on puisse comprendre les paroles et à qui on puisse attribuer celles-ci. C'est ici que se trouve le pivot de la théorie de Shabestarî. La compréhension d'un langage par des êtres humains est régie par une règle à laquelle nul ne peut se soustraire, fût-il prophète : dans un contexte historique donné, des paroles ne peuvent être intelligibles par une société humaine que si le locuteur est luimême humain ${ }^{70}$.

Le principe de l'intelligibilité du langage a des conséquences méthodologiques. Suivant les termes de l'auteur, puisque le Coran était la parole du Prophète, celui-ci avait un lien verbal humain avec les interlocuteurs de ce texte. En conséquence, on peut utiliser pour étudier le Coran toutes les méthodes qui sont employées pour étudier les œuvres en langue humaine: philosophie du langage, linguistique, nouvelle herméneutique, critique historique. «Non seulement il n'y a pas d'obstacle doctrinal à une telle tâche, mais il n'y a pas d'autre voie ${ }^{71} »$.

Malgré cette assurance, l'auteur sait qu'il touche ici un des points d'achoppement les plus cruciaux de la théologie musulmane. C'est pourquoi il n'a de cesse de préciser son intention dans les articles suivants. Dans l'article 2, il récapitule les thèses essentielles qu'il a formulées dans son article inaugural : «L'article "Lecture prophétique du monde" qui est paru dans le numéro 6 de la revue Madreseh n'est pas une théorie philosophique ni théologique ni scientifique sur la nature [haqîqat] de la parole divine, sur la révélation ou sur l'expérience prophétique et autres sujets semblables. Cet article est une théorie sur la manière de comprendre le Coran qui comporte deux thèses essentielles ». Nous reviendrons plus loin sur la formulation de ces thèses et sur leur portée. Il importe ici d'observer que l'auteur utilise sa méthodologie comme une prétérition. Suivant ses termes, la première thèse est liée à «l'herméneutique philosophique contemporaine » au sens général et «qui s'oppose à l'herméneutique dogmatique », tandis que la seconde thèse est liée à l'étude de la littérature. La première thèse ne prolonge pas la querelle ancienne des théologiens sur les termes et les sens du Coran, ni sur sa nature. C'est une nouvelle thèse

70. Shabestarî, Qerâ'at-e nabawî az jahân, nº 1,16 bahman 1386 / 5 février 2008.

71. Ibid. 
herméneutique, philosophique, qui se préoccupe de la réalité de la compréhension et de ses modalités. Les conceptions du Coran des théologiens passés n'ont pas été exposées selon le point de vue de l'herméneutique philosophique contemporaine. Or on ne peut tendre à une compréhension du Coran, qui est un texte en langue arabe, en négligeant les données de la philosophie contemporaine du langage. Avant de faire l'exégèse du Coran, il faut donc adopter un point de vue sur celles-ci. Shabestarî précise que l'une des plus importantes hypothèses préalables à l'exégèse d'un texte consiste en ce que le locuteur réel doit être un être humain réel. Il ajoute qu'il entend par compréhension d'un texte une compréhension universelle. Cela signifie qu'un croyant aussi bien qu'un noncroyant peut accéder à une compréhension du texte. Car ce qui ne peut être compris universellement ne peut être compris sous aucune forme. Au contraire, si l'on suppose que le locuteur est Dieu même, on rend le texte coranique incompréhensible pour un non-croyant ${ }^{72}$.

Un peu plus loin dans l'article 2, l'auteur réitère son propos. Il insiste sur le fait que dans tous ses travaux antérieurs il a cherché à «élargir la voie pour comprendre et interpréter le texte du Livre [le Coran]», et non à théoriser l'expérience prophétique ou la parole révélée. Il a certes, concède-t-il, parfois qualifié la révélation d'expérience prophétique, notamment dans un chapitre de sa Critique d'une lecture officielle de la religion ${ }^{73}$. Malgré cela, assure-t-il, la Lecture prophétique du monde n'est pas « une théorie sur la nature de la parole de Dieu». Mais «on peut comprendre à partir du Coran que la révélation a été le motif pour lequel le Prophète a parlé dans le $\operatorname{Livre}^{74}[\ldots]$ ». Sans craindre d'être redondant, Shabestarî clarifie son approche. Dans l'article 3 intitulé «Hypothèses préalables de l'exégèse libre du Coran. 2. Réquisits de la compréhension et réquisits de la foi », il énumère les différentes questions qui ont été soulevées à propos des textes scripturaires et les disciplines dans le cadre desquelles elles ont été

72. Shabestarî, Qerâ'at-e nabawî az jahân, nº 2, 22 bahman 1387 / 10 février 2009.

73. L'auteur mentionne le titre du chapitre, «La beauté de la parole de Dieu et l'élargissement de l'horizon humain ». Cf. Naqdî bar qerâ'at-e rasmî az dîn, Téhéran, Tarh-e nô, (1379 AHS/2000), 4édition 1390/2011.

74. Shabestarî, Qerâ'at-e nabawî az jahân, $\mathrm{n}^{\circ} 2$. 
traitées. La question de savoir si Dieu crée la parole ou s'il parle relève de la théologie et de la philosophie. Il en va de même de la question relative à la réalité des événements évoqués dans les révélations faites aux prophètes. D'autre part, les interprétations faites par les juifs, les chrétiens et les musulmans de leurs textes scripturaires concernent l'histoire de l'exégèse. Enfin, une quatrième question porte sur la valeur que les exégèses anciennes ont à l'époque actuelle du point de vue philosophique, scientifique ou théologique. C'est cette question seulement que Shabestarî entend aborder. Il s'interroge sur les hypothèses préalables que l'on peut formuler à l'époque actuelle pour faire une exégèse rationnelle universelle du Coran qui est un texte en langue arabe. Avant d'expliciter sa propre hypothèse, il rappelle l'hypothèse préalable prédominante qui sous-tend l'exégèse du Coran. Selon cette hypothèse, qui est théologique, le Coran a été donné par Dieu au prophète de l'islam littéralement. Sans aucun acte personnel, le prophète Muhammad aurait récité le Coran à ses interlocuteurs. L'auteur annonce qu'il veut critiquer cette hypothèse car elle empêche la compréhension du Coran considéré comme parole ${ }^{75}$.

Il lui incombe alors de définir la compréhension et ses réquisits. Shabestarî consacre le troisième article à cette question, en indiquant qu'il en tirera ensuite les conclusions. Ainsi il renverse l'ordre de l'argumentation qui préside à l'exégèse théologique du Coran en islam. Selon lui, c'est l'approche herméneutique qui est primordiale et qui a des corrélats théologiques, et non l'inverse. L'auteur analyse donc le processus de la compréhension en se concentrant sur la communication langagière. Il le décrit de manière didactique et en détaille les étapes. De cet examen il tire une conséquence capitale pour son propos : c'est seulement dans le monde humain qu'une parole peut être universellement comprise. $\mathrm{Si}$ au contraire on suppose qu'une réalité qui ne se produit pas dans le monde humain a un sens, il faut s'interroger sur le sens de cette réalité. En d'autres termes, il faut un locuteur humain. Dès lors que l'on admet que le Coran est compris comme la parole d'un locuteur, force est de dire que ce texte était la parole du prophète de l'islam. Du point de vue de la compréhension, insiste Shabestarî,

75. Shabestarî, Qerâ'at-e nabawî az jahân, no 3, 25 bahman 1387 / 13 février 2009. 
les musulmans ne peuvent rien admettre sinon que le Coran est la parole de Muhammad. Car tel est le réquisit de la compréhension rationnelle. Shabestarî assure qu'il ne peut ni réfuter ni corroborer les doctrines exposées par les philosophes, les mystiques et les théologiens musulmans à propos de la révélation muhammadienne. Mais il ne peut admettre que l'on puisse soutenir que le Coran est incompréhensible pour les hommes. Or, affirme-t-il, la doctrine théologique en vigueur en islam rend le Coran incompréhensible pour les hommes ${ }^{76}$.

\section{Le Coran, « expérience herméneutique » du prophète de l’islam}

Malgré les précautions méthodologiques dont il use, l'hypothèse herméneutique conduit bel et bien Shabestarî à définir l'identité de l'auteur du Coran. Celle-ci établie, le théologien recourt à l'herméneutique pour qualifier le statut et le style du texte révélé. Qu'il s'agisse de la création du monde et de l'homme, des phénomènes naturels et de la vie humaine, les versets coraniques expriment une vision prophétique qui est une «interprétation » ou « lecture »: « Outre les phénomènes naturels, la destinée et la mort de l'homme et l'histoire des peuples, le texte du Coran expose un essai d'interprétation prophétique de la vie sociale des gens du Hijâz. Les principes moraux et les préceptes religieux (sur les cultes et les transactions) du Coran résultent de cette expérience. » Les versets sont la base de la vision du prophète de l'islam sur la destinée de l'homme ${ }^{77}$. Ce qui importe, ajoute l'auteur, c'est de prendre en considération le fait que le jugement sur les relations entre les hommes ou entre les hommes et Dieu prend sens dans une société déterminée et s'apparente toujours à une interprétation. Shabestarî illustre son propos en se référant aux versets juridiques du Coran. À l'appui de la notion d'interprétation, il redéfinit en fait la nature et la portée des «préceptes » ou «jugements ». Selon lui, il est possible que tous les préceptes aient été appelés «jugements » (hokm) parce qu'ils comportent tous un jugement (qadâwat) sur quelque chose. Tout ceci prouve clairement que les versets juridiques du Coran reposent sur une interprétation

76. Shabestarî, Qerâ'at-e nabawî az jahân, $\mathrm{n}^{\circ} 3$.

77. Shabestarî, Qerâ'at-e nabawî az jahân, $\mathrm{n}^{\circ} 1$. 
des réalités et des relations sociales dans le Hijâz, ainsi que des relations entre les hommes et Dieu. «La formulation de ces préceptes ne signifie absolument pas que des préceptes ont été établis pour toutes les sociétés et pour toutes les époques [...] Du texte du Coran, on n'obtient que ce que nous avons expliqué. » À l'encontre des philosophes musulmans, Shabestarî soutient que les versets coraniques n'exposent pas des prédicats métaphysiques mais "manifestent l' "expérience herméneutique" du monde faite par le Prophète ${ }^{78} »$. Dans l'article 2, il réfute plus explicitement le dogme de la nature du Coran. À l'appui de certains versets présentant le Coran comme un livre révélé, les théologiens musulmans considèrent que l'on ne peut pas accepter la thèse selon laquelle le Coran est une parole prophétique. L'auteur leur répond en trois points. Premièrement, on ne peut comprendre les versets qu'ils citent que si l'on admet qu'ils sont une parole humaine. Il faut préciser, exige-t-il, que le Prophète dit que le Coran est un livre révélé. Deuxièmement, il faut chercher ce que peut signifier une telle affirmation du Prophète. En recourant à la fois à certains versets, à la culture arabe antéislamique et aux précédents analogues dans le judaïsme et dans le christianisme, on peut comprendre que pour Muhammad les versets prenaient forme avec l'aide de la révélation. Selon Shabestarî, le Coran lui-même prétend être «le résultat de la révélation et non la révélation même ». Troisièmement, il n'est dit dans aucun verset que le Coran n'est pas la parole de Muhammad. De surcroît, le texte coranique lui-même indique clairement qu'il y avait entre ce dernier et ses interlocuteurs - croyants et incroyants - des débats, des accords et des désaccords. Ceci signifie que les incroyants ont également compris le contenu des versets du Coran et que les «revendications » $\left(\bmod d a^{c} \hat{a} t\right)$ du texte étaient comprises de ceux qui l'entendaient ${ }^{79}$. Du point de vue de la foi, les musulmans peuvent croire que Muhammad a exprimé les paroles coraniques sous l'influence d'une parole directe ou indirecte adressée par Dieu. Mais si l'on fait abstraction de la foi, il apparaît que le Prophète a fait l'expérience d'être l'interlocuteur de Dieu ${ }^{80}$. On peut donc

78. Shabestarî, Qerâ'at-e nabawî az jahân, $\mathrm{n}^{\circ} 1$.

79. Shabestarî, Qerâ'at-e nabawî az jahân, $\mathrm{n}^{\circ} 2$.

80. Shabestarî, Qerâ'at-e nabawî az jahân, $\mathrm{n}^{\circ} 2$. 
établir une hypothèse recevable par des croyants autant que par des non croyants : ce que le Prophète éprouve comme parole de Dieu est effectivement parole de Dieu pour lui. Cette hypothèse est bien différente de celle d'après laquelle le contenu de l'expérience du Prophète est lui-même parole divine ${ }^{81}$. « [...] Si l'on est encore plus précis, affirme plus loin Shabestarî, on sera conduit à la conclusion que la parole non humaine de Dieu qui, par hypothèse, parvient au Prophète, n'est en aucune manière susceptible d'être transmise. »Car on ne peut «transmettre » que ce qui est susceptible d'être compris dans le monde humain intersubjectif. Or une parole non humaine n'a pas ces caractéristiques. En conséquence, il faut admettre «l'hypothèse qui est non seulement intelligible mais inévitable », selon laquelle «ce texte [le Coran] est "la parole prophétique" d'un être humain ». Il faut considérer finalement que le Prophète lui-même est «l'auteur de la parole » (sâheb-e kalâm). Et Shabestarî renvoie en note aux Investigations philosophiques de Wittgenstein ${ }^{82}$. Plus explicitement encore, il affirme dans l'article 11: "C'est le prophète de l'islam qui a fait apparaître dans l'histoire le Coran sous la forme d'un texte». Sans ces actes de langage de Muhammad, il n'y aurait pas de texte appelé Coran ${ }^{83}$.

L'auteur souligne en outre que son hypothèse a des implications exégétiques. En effet, si l'on considère le Coran comme la parole du Prophète, on fait "l'exégèse d'un texte historique survenu dans le tissu de la vie historique, culturelle et sociale des hommes [...] On peut ainsi élaborer des exégèses totalement différentes de celles qui reposent sur le postulat de la nature divine du Coran ${ }^{84}$. »

Dans l'article conclusif intitulé «Qu'est-ce que le Coran?» (qor'ân chîst), Shabestarî recourt à l'herméneutique pour mettre en évidence le caractère narratif du texte coranique : «Je soutiens que si l'on prend en considération les philosophes modernes du langage et l'herméneutique moderne, comprendre le Coran comme un

81. Shabestarî, Qerâ'at-e nabawî az jahân, no 5, 28 bahman 1387 / 16 février 2009.

82. Shabestarî, Qerâ'at-e nabawî az jahân, nº 7, 27 bahman 1388 / 16 février 2010.

83. Shabestarî, Qerâ'at-e nabawî az, jahân, n 11, 6 khordâd 1391 / 26 mai 2012.

84. Shabestarî, Qerâ'at-e nabawî az jahân, n ${ }^{\circ} 6,1^{\text {er }}$ esfand 1387 / 19 février 2009. 
récit est la compréhension et l'exégèse la plus défendable ${ }^{85} \gg$. De manière didactique, l'auteur définit le texte et la «compréhension narrative » (narrative Verstehen dans le texte) en vue de démontrer que le Coran ne décrit pas Dieu mais livre un récit sur Dieu. Selon lui, le récit coranique se divise en trois parties : récits sur le commencement (ou création), sur la suite et sur la fin. Les trois catégories de récit constituent «la charpente du texte du Coran ». Finalement, en se référant à Temps et Récit de Ricœur, le théologien récapitule son étude. Le Coran est une compréhension narrative exprimée dans le langage de la métaphore. Or « on ne peut parler de Dieu qu'avec le langage de la métaphore ${ }^{86} »$.

\section{Conclusion}

Si les pratiques exégétiques islamiques ont été marquées depuis le $\mathrm{XIX}^{\mathrm{e}}$ siècle par les savoirs étrangers, principalement européens, Shabestarî incarne un tournant décisif dans l'histoire de la pensée islamique et de ses échanges avec la philosophie européenne moderne. Jusqu'alors, à de rares exceptions près, les auteurs musulmans qui se référaient à celle-ci étaient empreints d'esprit apologétique. De plus, la philosophie européenne et américaine moderne était restée largement méconnue en Islam jusqu'à la fin $\mathrm{du} \mathrm{XX}^{\mathrm{e}}$ siècle. En introduisant l'herméneutique philosophique, Shabestarî instaure de nouveaux rapports tant avec la tradition islamique qu'avec la philosophie européenne. Son recours à l'herméneutique nourrit d'abord une critique épistémologique radicale des disciplines du savoir religieux classique. Il lui permet ensuite de repenser la nature du texte coranique. En effet, bien qu'il souligne la visée méthodologique de son "exégèse libre du Coran », le clerc en tire des conclusions théologiques explicites. Il conteste le dogme de l'origine divine du texte sacré et considère celui-ci comme une « expérience herméneutique » du prophète Muhammad ${ }^{87}$.

85. Shabestarî, Qerâ'at-e nabawî az jahân, no 15, 17 mordâd 1392 / 8 août 2013.

86. Shabestarî, Qerâ'at-e nabawî̀ az jahân, $\mathrm{n}^{\circ} 15 / 2$, «Article conclusif. Derniers mots », 21 mordâd 1392 / 12 août 2013.

87. L'argumentation de Shabestarî est trop claire pour que l'on puisse admettre avec Ali Akbar qu'elle «ne sape pas le statut divin du texte du Coran ». Cf. Akbar, "A Contemporary Muslim Scholar's Approach to Revelation: Mohammad Mojtahed Shabestarî’s Reform Project », article cité, p. 680. 
Dans ses efforts pour acclimater l'herméneutique, Shabestarî se distingue autant par son exhaustivité que par son éclectisme. Il fait converger des lignées philosophiques distinctes dans le but principal de récuser l'approche métaphysique des textes scripturaires. Enfin, quoique cette dimension n'ait pas été examinée ici, il importe de considérer que le théologien conjugue l'approche critique des savoirs et des textes à une herméneutique de l'expérience religieuse qui lui permet d'élaborer une théologie nouvelle.

constance.arminjon@ephe.psl.eu 